\title{
Capital financiero versus complejo médico-industrial: los desafíos de las agencias regulatorias
}

\author{
Financial capital versus medical-industrial complex: \\ challenges for the regulatory agencies
}

${ }^{1}$ Department of Family and Community M edicine, Senior Fellow RWJF Center for Health Policy, University of $\mathrm{N}$ ew M exico.

Albuquerque, NM 87131

USA. ciriart@salud.unm.edu
Abstract This article presents the structural processes that consolidated under the hegemony of the financial capital in the 90s; the dispute be tween the financial capital operating in the health sector and the medical-industrial complex; the strategies used by the medical-industrial complex for regaining positions; and the challenges all these processes pose for the regulatory agencies. The problems the regulatory agencies are facing lie in two central processes: 1) the hegemony the financial capital reached in the 90s in the health sector through reforms aimed at deregulating the sector in order to facilitate its entrance; and 2) the repositioning of the medical-industrial complex since the mid 90 s by radicalizing medicalization. This article is based on several studies conducted by the author using qualitative methods and quantitative secondary data for understanding the historical-situational context. The theoretical approach was based on M arx, Gramsci, Benasayag, Badiou, Testa and M erhy. The analyses of the most recent reforms induced by the medical-industrial complex were the result of a bibliographic and document review.

Key words H ealth sector reform, Latin America, Financial capital, M edical-industrial complex, Distribution dispute, Regulation
Resumen Se presentan los procesos estructurales consolidadosen los 90s bajo la hegemonía del capital financiero; las pujas distributivasgeneradas con el complejo médico-industrial; las estrategias que éste usó para reposicionarse; y los desafíos que enfrentan lasagencias regulatorias estatales. D os procesos son fundamentales para ubicar la problemática que enfrentan las agencias regulatorias: 1) La hegemonía alcanzada en los 90s por el capital financiero en el sector salud, vía las reformas destinadas a desregularlo; y 2) el reposicionamiento del complejo médico-industrial desde mediados de los 90 s, a través de radicalizar la medicalización. Este artículo se basa en varias investigaciones conducidas por la autora en las que se usaron métodos cual itativos y datos secundarios cuantitati vos para la contextualización histórico-situacional. El abordajeteórico se basó en M arx, Gramsci, Benasayag, Badiou, Testa y M erhy. El análisis de las reformas más recientes impulsadas por el complejo médicoindustrial es producto de una investigación bibliográfica y documental.

Palabras clave Reforma del sector salud, América Latina, Capital financiero, Complejo médicoindustrial, Puja distributiva, Regulación 


\section{Introducción}

La regulación estatal en el campo sanitario confronta nuevos y complejos desafíos. Las reformas sectoriales introducidas en los 90 s, como parte de las transformaciones operadas en el capitalismo mundial bajo la hegemonía del capital financiero se han mantenido y profundizado en la década del 2000.

En este artículo me propongo presentar los procesos estructurales que se consolidan en los 90 s bajo la hegemonía del capital financiero; las pujas distributivas que se generan con el hasta entonces casi indiscutido actor central del sector salud, esto es el complejo médico-industrial; las estrategias que éste usó para reposicionarse; y los desafíos que esto implica para las agencias regulatorias estatales.

Los procesos que quiero destacar, si bien son instituidos en y desde los países centrales, en especial los Estados Unidos, tienen implicancias fundamentales para los países latinoamericanos, ya que afectan el proceso regulatorio estatal, las disputas intercapitalistas en el sector salud a nivel de los países; y las estrategias que los grupos que cuestionan el modelo hegemónico adoptan.

Dos son los procesos que considero fundamentales paraubicar la problemática queenfrentan las agencias regulatorias y otros actores sociales interesados en generar políticas desalud en favor dela vida individual y colectiva. Estos procesos son: 1) La hegemonía alcanzada en los 90s por el capital financiero en el sector salud, a través de las reformas destinadas a desregularlo para facilitar su entrada masiva; 2) El reposicionamiento del complejo médico-industrial desde mediados de los 90s, a través de radicalizar la medicalización, creando estrategias de comunicación dirigidas directamente a los usuarios, cambiando la definición de enfermedades y creando nuevas entidades nosológicas; presionando a las agencias regulatorias para aprobar nuevos fármacos, tecnologías y tratamientos para que sean financiados por los seguros de salud públicos y privados; y la difusión y generalización de los protocolos clínicos y de la medicina basada en la evidencia.

\section{Material y métodos}

Este artículo se basa, para la parte del análisis de las reformas impulsadas por el capital financiero en los resultados de varias investigaciones en las cuales participé como investigadora principal o asociada ${ }^{1-4}$. En estas investigaciones se usaron métodos cualitativos de recolección de datos, especialmente, entrevistas en profundidad a informantes claves, y recopilación documental y bibliográfica. Para analizar el material recolectado utilizamos análisis de discurso y documental. Asimismo, seanalizaron datos secundarios cuantitativos para la contextualización histórico-situacional. El análisis de las reformas más recientes impulsadas por el complejo médico-industrial son producto de una investigación bibliográfica y documental. El proceso analítico sebasa en triangulación metodológica con un abordaje teórico que incluye las contribuciones de M arx, Gramsci, Benasayag y Badiou en lo que se refiere a la comprensión del campo de la organización de las prácticas sociales y políticas contemporáneas, con sus contradicciones y disputas exacerbadas por el actual proceso de intensa transformación social generada por la dinámica global delas sociedades hegemónicas. Así como de autores latinoamericanos, en especial, Testa y M erhy, en los temas referidos al poder y la micropolítica del trabajo en salud.

Hegemonía del capital financiero en el sector salud

El primer proceso que quiero destacar es el que se refiere a la hegemonía que adquiere el capital financiero en la mundialización del capitalismo desde la crisis de mediados de los 70s, conocida como crisis del petróle ${ }^{5}$. En este período el capital productivo ve limitada su expansión por crecimiento de los costos de las materias primas y de los salarios. Los dueños del capital mueven sus inversiones desde el sector productivo hacia los mercados financieros y en muchos casos hacia los mercados offshore ${ }^{6}$.

El mayor crecimiento del capital financiero se registra entre los fondos mutuales y de pensión, y el capital generado por las actividades ligadas a los seguros. En Estados Unidos entre 1980 y 1993 estos fondos crecieron de 1.606 millones de dólares a 8.008 millones. Los fondos de pensión de los Estados Unidos representaban en 1994 el $82,7 \%$ entre los cinco principales países que los generaban. Otro dato para entender la importancia queadquiereel capital financiero en la economía capitalista es que las ganancias de los administradores de fondos mutuales y de pensión y de los agentes de bolsa pasaron de 4.800 millones de dólares en 1975 a 69.500 millones en 1994. A manera de comparación cabe señalar que M i- 
crosoft, una de las empresas más exitosas del mundo, obtuvo una ganancia de "solo" 2.200 millones de dólares en 19947. Estos datos son importantes porque es en la primera mitad de la década del 90 , que las reformas en el sector salud lideradas por el capital financiero se generalizan. Esto no quiere decir que este capital no operaba con anterioridad en el sector salud, sino que se torna la fuerza que hegemoniza las reformas.

Estas cantidades crecientes de capital financiero requirieron de nuevos países y sectores dentro de los países donde reproducirse. Esto demandó cambios anivel político, a través de un proceso de desregulación - privatización - reregulación, así como de profundos cambios ideológicos. A nivel ideológico, el neoliberalismo seinstala como pensamiento único y en salud implica la transformación del sentido común para que la salud deje de ser un derecho que el estado debe garantizar y se transformeen un bien de mercado que los individuos deben adquirir. La lógica privada permea todos los espaciosy el sector público "debe" adoptarla para operar eficientemente. A nivel político la desregulación permite al capital financiero internacional operar en el sector salud de los países, antes prohibido total o parcialmente ${ }^{4,8}$.

En este contexto surgen las llamadas organizaciones de atención gerenciada o $\mathrm{M}$ anage $\mathrm{Care}$ Organizations (MCOs) por su nombre en inglés. Estas son empresas de lucro creadas como subsidiarias por las empresas de seguro y por las administradoras de fondos mutuales y de pensión, para entrar en la administración de fondos de salud públicos y privados, primero en los Estados Unidos y luego en el resto del mundo,10. En los Estados Unidos, hacia mediados de los 90s, las empresas de atención gerenciada obtuvieron la desregulación de los programas públicos de salud (M edicaid y M edicare), para entrar como administradoras de estos fondos públicos y como contratadoras de servicios de atención médica para los afiliados quese inscribían en ellas. Desde comienzos de los 90s se convirtieron en las administradoras de elección de los seguros provistos por los empleadores dejando en un segundo plano a las denominadas $\mathrm{H}$ ealth M aintenance O rganizations (H M Os) ${ }^{11,12}$. En este punto me parece importante aclarar la diferencia entre los términos HMO y MCO, ya que es muy común que se usen erróneamente como sinónimos. Las HM Os obtuvieron un gran impulso en los Estados Unidos bajo la administración Nixon, que en 1973 logra la aprobación dela H ealth M aintenance Organization Act. Las HM Os se crearon como una alternativa a los seguros indemniza- torios y debían cumplir con tres requisitos básicos: ofrecer una lista de beneficios a todos los miembros por igual, cobrar la misma mensualidad a todos los asociados, y no tener fines de lucro ${ }^{13}$. Las MCOs, creadas por el capital financiero hacia los 90s, son empresas con fines de lucro cuyo negocio es administrar los recursos financieros destinados a la compra de servicios para la cobertura médica ofrecida por empleadores y por programas públicos o semipúblicos.

A nivel ideológico la atención gerenciada gana adeptos con el argumento de que van a reducir costosvía el aumento de prácticas preventivas y el uso racional de recursos de diagnóstico y tratamiento, basándoseen la evidencia científica. El eje central del discurso dela reforma bajo la atención gerenciada era instituir el médico deatención primaria como un agente coordinador de la atención y moderador de la utilización de atención especializada, así como de diagnósticos y tratamientos costosos y de no probada eficacia ${ }^{14}$. Sin embargo, ya en 1997 en los Estados Unidos, las empresas de atención gerenciada tenían: 8\% menos beneficiarios con inmunizaciones completas que las organizaciones sin fines de lucro; $6 \% \mathrm{me}$ nos con mamografías; 8\% menos con papanicolau; $12 \%$ menos con prescripción de betabloqueantes en pacientes que habían sufrido ataques al corazón; y 13\% menos con controles oftalmológicos en pacientes diabéticos. La situación incluso puede que haya sido peor que lo reflejado en los datos, ya que estos son reportados en forma voluntaria por las empresas y no auditados ${ }^{15}$.

La realidad es que más allá de los discursos y promesas con la que la atención gerenciada logró posesionarse, lo que se evidenció en pocos años desdesu implementación esqueen la administración de fondos de salud el capital financiero obtiene ganancias limitando acceso y controlando gastos. Deallí quelos médicos deatención primaria cumplen bajo los contratos con las M COs un papel de controladores de la demanda y no coordinadores de la atención. La operatoria de este tipo de capital requiere dela transformación, casi constante de los planes de salud ofrecidos, como forma de dificultar el acceso y disminuir el gasto. Cuando los beneficiarios aprenden a navegar el complejo sistema administrativo y sortean las dificultades y "errores" burocráticos en la asignación del médico de atención primaria, aprobación de estudios y tratamientos, reintegros, etc., los contratos dejan de ser rentables para las M COs. Esto sucedeen el transcurso de tres o cuatro años. Asimismo, las regulaciones impuestas poco a poco por los entes federales y estatales 
como respuesta a las demandas de los usuarios, de las asociaciones de consumidores y de profesionales, crearon una limitación para las ganancias delas organizaciones de atención gerenciada. Así fue como hacia la segunda mitad dela década del 90 las M CO s se expandieron hacia otros países para aumentar las opciones de ganancias y negociaron en los Estados Unidos con la administración federal, para entrar en la administración de los fondos de M edicare y con los gobiernos estaduales para administrar los fondos de M edicaid. Al cumplirse el ciclo de ganancias en el sector salud se observa la misma volatilidad que en otras áreas donde el capital financiero invierte. Al disminuir las ganancias el capital emigra a otras regiones 0 áreas de la economía. Hacia fines del 2000, CIGNA y Aetna, dos de las empresas de seguro más grandes de Estados Unidos, dejaron de administrar los fondos de M edicare en 11 y 13 estados respectivamente. Entre 1998 y 2002, las salidas de las M COs de los contratos con M edicare afectaron 2.200 .000 beneficiarios $^{16}$.

En América Latina procedieron de forma similar, muchos países desregularon sus economías para facilitar la entrada de aseguradoras multinacionales en el sector salud. El capital financiero entró vía las empresas de seguro y las administradoras de fondos mutuales y de pensión de los Estados Unidos que derivaban los fondos captados en este país hacia mercados offshore, donde empresas demuy dudoso origen y finalidad (como el Grupo Exxel de Argentina) los captaban e invertían en el sector salud de países latinoamericanos. En Argentina operaron a través de la compra o asociación con empresas que venden planes prepagados de salud y desde allí participaron de la reforma de las obras sociales. En este sentido negociaron con los dirigentes de estas instituciones contratos para ser los administradores y contratadores de los servicios de aten ción médica de los beneficiarios. Las estrategias de comercialización de las M COs apuntaron a los trabajadores jóvenes, sanos, sin familia o con familia reducida y con altos salarios 4 . En Brasil, operaron desde el sector suplementar comprando empresas de grupo y aseguradoras, para entrar en la administración de los planes de salud comprados por individuos y empresas para sus empleados. Es importante destacar que al capital financiero le importa fundamentalmenteentrar en la administración de fondos públicos y de la seguridad social o de grupo, ya que el mercado privado dirigido a individuos es muy pequeño en todos los países.

$\mathrm{H}$ acia fines de los 90 s y comienzos de la década del 2000 las empresas multinacionales de ori- gen estadounidense salieron de la mayoría de los países latinoamericanos. Sin embargo, su salida no cambió, en muchos de nuestros países, el curso que el sector salud tomó en los 90s. Gerenciadoras y empresas comercializadoras de planes de salud nacionales tomaron su lugar con prácticas similares o peores. El capital financiero operando como instituyente de la reforma neoliberal había logrado su objetivo ${ }^{17}$. La lógica privada, el mercado como regulador cuasi soberano, la competencia entre servicios estatales y entre estos y los privados, la eficiencia mirada desde las instituciones individuales y no desde el sistema y menos desde los usuarios y trabajadores, penetró el sector estatal, la seguridad social y los seguros ofrecidos a sus trabajadores por las empresas ${ }^{18}$.

El capital financiero también entró en la administración de servicios de atención de salud. Grupos financieros compraron hospitales, residencias para pacientes crónicos o que requieren cuidados médicos prolongados (nursing homes), y otro tipo deservicios. Lo queel capital financiero hace es administrar los espacios y recursos físicos cobrando por su utilización a grupos profesionales deprácticas independientes instalados en dichos establecimientos. Esto establece una cadena de clientes internos entre los servicios y es una situación que puede ser interpretada y bienvenida por al gunos profesionales como la revitalización dela concepción liberal, pero solo setrata de una forma de eliminar la relación laboral entre administradores y profesionales, y de poner el peso de la responsabilidad legal y financiera en estos últimos. Esta práctica absolutamente generalizada en los Estados Unidos, se ha instalado también en América Latina, en especial en los grandes hospitales privados ${ }^{19}$.

\section{Reposicionamiento del complejo médico-industrial}

El proceso antes descripto implicó la instalación deuna puja por los recursos económicos entreel capital financiero y el capital del llamado complejo médico-industrial. El negocio del capital financiero es extraer recursos económicos del sector salud para realizar las ganancias de los inversoresy para invertir en áreas más rentablescuando la oportunidad aparece. Esta práctica sumada a la restricción impuesta al acceso a los servicios de salud para controlar el gasto, chocó con los intereses del capital ligado a empresas productoras de tecnologías y medicamentos, y de los propios profesionales de salud. 
En este punto, una nueva pregunta redireccionó mis análisis como resultado de profundizar en el estudio de las reformas sectoriales hegemonizadas por el capital financiero, así como en el del sistema de salud de los Estados Unidos y en los procesos de reforma sectorial allí impulsados. El capital industrial había tenido en los profesionales de salud, en especial en los médicos, a sus aliados centrales para aumentar la demanda de sus productos y a ellos dirigieron por años sus estrategias informativas ${ }^{20}$. De allí que también fueron los médicos el centro de la estrategia de control administrativo desarrollada por las MCOs. Limitada esta fuente de creación de demanda, la pregunta que se imponía era ¿qué acciones estaban tomando las farmacéuticas y empresas productoras de tecnología, para contrarrestar las re formas desarrolladas por el capital financiero?

Durantela investigación sobrela exportación dela atención gerenciada que condujejunto a un equipo internacional a mediados de los $90 \mathrm{~s}$, emergen los primeros datos de como las compañías ligadas al complejo médico industrial negociaban con la seguridad social y con los seguros privados, para que estas instituciones modificaran sus formularios terapéuticos incluyendo productos de alta rentabilidad para las compañías farmacéuticas y presionaban a los entes regulatorios para que aprueben nuevos estudios y tratamientos con la finalidad de que fueran financiados por los seguros ${ }^{21}$. Asimismo, compañías farmacéuticas y de tecnología compraron o hicie ron acuerdos con cadenas de hospitales especializados, centros de diálisis, redes de emergencias, y otros centros de atención especializada, para asegurarse nichos de consumo de sus productos ${ }^{22}$. También, algunas empresas crearon y/0 apoyaron con equipamientos, insumos y medicamentos a fundaciones que proveen servicios en los hospitales estatales. Estas fundaciones surgieron en los 90s como parte de los acuerdos que en al gunos países el sector gubernamental realizó con el sector privado, para que provean servicios dediagnóstico y tratamiento en hospitales públicos, cobrando por los servicios a los pacientes, a la seguridad social y/o a los seguros privados. La administración estatal recibía como compensación por el uso de las instalaciones, costos de funcionamiento y, en algunos casos, incluso insumos y personal, una cantidad de prestaciones mensuales para los pacientes sin capacidad de pago, mientras que las empresas se aseguraban el consumo de sus productos ${ }^{18}$.

Diversos autores anglosajones están documentando otros fenómenos que se suman a los anteriores ${ }^{23}$. Desdela segunda mitad de los 90 s se vienen operando importantes transformaciones que son parte de este proceso de puja distributiva y que están originadas en la presión de la industria farmacéutica y de tecnología por generar regulaciones legales y normativas científicas a favor de sus intereses comerciales. Muchos de estos cambios se hacen en el ámbito de comités de expertos reunidos por agencias estatales $u$ organismostécnicos internacionales, y en universidades, principalmente delos Estados Unidos, y sus decisiones, amparadas en la lógica científica dominante, son tomadas como normas universales. En este contexto se redefinieron ciertas enfermedades, se transformaron riesgos en enfermedades y nuevas nosologías fueron definidas ${ }^{24}$. Protocolosy medicina basada en la evidencia se transformaron en reglas a seguir y palabras a invocar para no quedar fuera del consenso científico hegemónico ${ }^{25,26}$. La aparición y generalización de la medicina basada en la evidencia, con sus seguidoras, las políticas, las intervenciones, y la salud pública basadas en la evidencia, así como la generalización de los protocolos clínicos reinstauraron la hegemonía de las corrientes biologistas, procedimiento centradas, individualistas, que habían sido puestas en cuestionamiento durante los $60 \mathrm{~s}$ y 70s por las corrientes de la medicina social/salud colectiva. Estos procesos sellaron el círculo virtuoso de la cientificidad bajo la hegemonía de una recreada biologización y medicalización.

Comités de "expertos" redefinieron en la segunda mitad de los 90s, el concepto de saludenfermedad de diversas nosologías y riesgos: El nivel de glucosa para determinar si una persona es diabética pasó de $>140 \mathrm{mg} / \mathrm{dl}$ a $>126 \mathrm{mg} / \mathrm{dl}$; la presión arterial alta de $>160 />100 \mathrm{mmH}$ ga $>140$ / >90 mmH g; la definición de hipercolesterolemia de $>240 \mathrm{mg} / \mathrm{dl} \mathrm{a}>200 \mathrm{mg} / \mathrm{dl} ;$ y el sobre peso medido como índice de masa corporal de $>27$ $\mathrm{kg} / \mathrm{m} 2 \mathrm{a}>25 \mathrm{~kg} / \mathrm{m} 2$. Estos cambios en la prevalencia de estas cuatro condiciones permitió, solo en los Estados Unidos, el siguiente aumento de casos: diabetes $14 \%$; hipertensión 35\%; hipercolesterolemia $86 \%$ y sobrepeso $42 \%$. Esto significó que 140.630 .000 personas adicionales ( $75 \%$ de la población adulta) pueden ser diagnosticadas con estos riesgos o enfermedades y sometidas a tratamiento ${ }^{24}$.

A estas enfermedades y riesgos redefinidos le tenemos que agregar la definición de nuevas enfermedades o la ampliación de diagnósticos ya establecidos para incluir más personas: la disfunción de erección y la consecuente promoción de medicamentos como Viagra27; la disfunción 
sexual femenina y deseo sexual hipoactivo y promoción de diferentes medicamentos incluido el Viagra off-label28; y el desorden premenstrual disfórico y promoción del uso deantidepresivos. En el caso del desorden bipolar, a la clásica definición de enfermedad maníaco-depresiva seagre gó el desorden bipolar no específico y la ciclotimia para ser medicadas con antipsicóticos ${ }^{29}$. $M$ ientras que en relación al déficit de atención e hiperactividad, las farmacéuticas promueven a través de revistas de divulgación y programas televisivosel aumento del papel depadresy maestros en el diagnóstico de los niños para aumentar la prescripción de psicoestimulantes ${ }^{30}$. Muchos de los diagnósticos se obtienen a través de cuestionarios autoadministrados ofrecidos en portales del nternet, revistas de divulgación, centros de atención primaria, escuelas, o enviados por correo postal a listas de personas que las compañías obtienen. Estos instrumentos consisten en una lista de síntomas y signos muy generales y subjetivos, de los que basta marcar algunos pocos para recibir la recomendación de consultar a un médico o contactar a la propia empresa farmaceútica para recibir más información.

En la actualidad, para expandir el mercado, los laboratorios no solo usan las conocidas tácticas demarketing, sino que, más y más prefieren financiar asociaciones de pacientes y familiares; publicidad directa de medicamentos bajo prescripción; publicidad encubierta usando campañas de "concientización"; compra de secciones en programas de televisión, incluyendo programas de noticias, uso de Internet y otras nuevas formas de comunicación; y campañas públicas con postas sanitarias ambulantes y ferias de salud para detectar población de riesgo y aumentar la demanda de servicios y en muchos casos de me dicación ${ }^{31-34}$.

A través de esto, millones de personas son blanco de este proceso medicalizador y medicamentalizador, etiquetadas como enfermas o en riesgo, y transformadas en consumidores al ertas para reclamar de los servicios de salud el último diagnóstico y/o tratamiento. Por otra parte, este proceso encubreque los problemas de salud más divulgados no tienen sus causas en desórdenes biológicos, sino en problemas sociales y políticos que quedan intocados ${ }^{35}$. Estos procesos están reforzando en los usuarios individuales una concepción de la salud-enfermedad-atención biologista, procedimiento centrada, basada en signos y síntomas individuales, que no analiza causas sino que diagnostica y trata efectos. Asimismo, están ejerciendo una enorme presión sobre los entes regulatorios para la aprobación de tratamientos con el objetivo de obtener la cobertura financiera del estado, de la seguridad social o de la medicina prepaga o suplementar, implicando un crecimiento de costos que restringe cada vez más el acceso a servicios realmente necesarios y de probada eficacia para las grandes mayorías ${ }^{36}$.

La salud pública a través de las nuevas corrientes anglosajonas, que también opera en los países latinoamericanos, contribuye a este proceso medicalizador y medicamentalizador, que culpabiliza a la víctima por comer en exceso, elegir alimentos no saludables, fumar, beber, estar estresado, etc. Esta "nueva" salud pública ha generado también, una promoción y prevención medicalizada, ya quetoda conducta saludable se basa en el control del riesgo de enfermar y en intervenciones individuales y procedimiento centradas como controlar el colesterol, la presión, la glucemia, los hábitos alimenticios, etc ${ }^{37}$. Los expertos en salud pública responsabilizan a los individuos por los malos hábitos que los llevan a enfermar y convertirse en un costo para el conjunto de la sociedad. M ientras los grupos de interés vinculados a las corporaciones ejercen presión sobre los gobiernos para evitar toda regulación y control sobre las actividades de las compañías productoras de comida chatarra, bebidas alcohólicas y gaseosas, productos transgénicos, y fármacos, tecnología einsumos médicos de escasa o nula eficacia, o lo que es peor muchas veces iatrogénicos. La ideología de la libertad de elección, el mercado como soberano, el individuo como último responsable y el estado como mero administrador opera eficientementepara producir lo quelascorporación en la lucha intercapitalista por aumentar las ganancias necesitan: consumidores "libres".

\section{Conclusiones}

La puja distributiva no se ha resuelto entre el capital financiero y el industrial, sino que ha adquirido nuevas formas que le dan a cada uno su parte, en desmedro del sector público, delos profesionales y de la salud de los colectivos sociales. La regulación ha adquirido una enorme complejidad, ya que las empresas creadas por el capital financiero utilizan numerosos artilugios jurídicos para encubrir su accionar fragmentándose en numerosas compañías sin aparente conexión y amparándose en las regulaciones creadas por los tratados de libre comercio. Esto les permite quedar exentas de responsabilidades frente a los usuarios y organismos regulatorios. Por su par- 
te, las empresas farmacéuticas y de tecnología han radicalizado el proceso medicalizador y medicamentalizador, a través de proveer información directa a los usuarios. Esto genera nuevas presiones sobre los profesionales de salud y mayores desafíos para las agencias regulatorias. Para enfrentar estos desafíos estas agencias deberían contar con el apoyo de expertos de diversos campos que analicen a diario la enorme cantidad de seudo información que la población recibea través de los medios de comunicación de todo tipo y con legislación adecuada que les permita san- cionar en tiempo y forma estetipo de manipulación informativa. Asimismo, el estado debería generar información de calidad para contrarrestar el impacto quela mala información produce. Asociaciones de profesionales deberían estar interesadas en analizar estos procesos y ponerse a la cabeza de movimientos sociales en defensa de la salud individual y colectiva. Esto redundaría en ben eficios para la práctica profesional que se está convirtiendo más y más en un mero engranaje del negocio de los grandes grupos que lucran con la salud.

\section{Referencias}

1. Iriart C, Waitzkin H, Merhy E, Estrada A, Breilh J Atención gerenciada en América Latina: su papel en la reforma de los sistemas de salud: Buenos Aires, Argentina: Instituto de Estudios y Formación/Central de Trabajadores Argentinos; 1999.

2. Waitzkin H, Iriart C. Global trade and U.S. health policies [proyecto de investigación]. Bethesda, $\mathrm{Ma}$ ryland: NIH/Agency for $\mathrm{H}$ ealth Care Research and Quality; 2003-2005.

3. Waitzkin $\mathrm{H}$, Iriart $\mathrm{C}$. M ental health services and global trade [proyecto]. Bethesda, M aryland: National Institute of M ental Health; 2003-2005.

4. Iriart C. The transnationalization of the health care system in Argentina. En: Mackintosh M, Koivusolo $M$, organizadores. Commercialization of health care: global and local dynamics, and policy responses. Hampshire: Palgrave Macmillan ( $\mathrm{M}$ acmillan Publishers Limited); 2005.

5. Foster JB. The financialization of capitalism. M onthly Review 2007; 58(11):1-12.

6. Bello W. Dilemmas of domination. The unmaking of the American empire. New York: Metropolitan Book Henry Holt and Co; 2005.

7. Petras J. La globalización: un análisis crítico. En: Saxe-Fernández J. Petras J, Veltemeyer H. Núñez 0 , editores. Globalización, imperialismo y clase social. Buenos Aires: Lumen; 2001.

8. Iriart C, Merhy E, Waitzkin H. Managed care in Latin America: the new common sense in health policy reform. Soc Sci \& M ed 2001; 52:1243-1253.

9. Waitzkin $\mathrm{H}$. The strange career of managed competition: from military failure to medical success? Am J Public Health 1994; 84(3):482-489.
10. Stocker $\mathrm{K}$, Waitzkin $\mathrm{H}$, Iriart $\mathrm{C}$. The exportation of managed care to Latin America. N Eng J M ed 1999; 340(14):1131-1136

11. Kuttner R. Must good HM Os go bad? First part. N Eng J M ed 1998; 338(21): 1558-1563.

12. Kuttner R. Must good HMOs go bad? Second part. N Eng J M ed 1998; 338(22):1635-1639.

13. Starr P. The social transformation of American medicine. New York: Basic Books; 1982.

14. Iriart $\mathrm{C}$. Atención gerenciada en América Latina: su papel en la reforma de los sistemas de salud. Salud, Problema y Debate 1999; 21:5-18.

15. Himmelstein D, Woolhandler S, Hellander I, Wolfe S. I. Quality of care in investor-owned vs not-forprofit HMOs. JAM A 1999; 282(2):159-163.

16. Iriart C, Waitzkin H, Merhy E. HMO's abroad: managed care in Latin America. En: Fort M, Mercer M, Gish O, editors. Sickness and wealth. The corporate assault on global health. Cambridge, MA: South End Press; 2004

17. Iriart C. Atenção gerenciada: instituinte da reforma neoliberal [tese]. Campinas (SP): Faculdade de Ciências M édicas; 1999.

18. Iriart $\mathrm{C}$, Waitzkin $\mathrm{H}$. Argentina: no lesson learned. Int J Health Serv 2006; 36(1):177-196.

19. Agência Nacional de Saúde Suplementar. Duas faces da mesma moeda: microrregulações e modelos assistenciais na saúde suplementar. Rio de Janeiro: ANS; 2005.

20. Kassirer J. On the take. How medicine's complicity with big business can endanger your health. New York: Oxford Press; 2005. 
21. Guerra J. La política de medicamentos en la década del '90 en Argentina. Salud, Problema y Debate 1997; 7(15):23-31.

22. Guerra J. Las corporaciones farmacéuticas y su mercado global. Salud, Problema y Debate 2000; 9(22):1-16.

23. Moynihan R, Henry D. The fight against disease mongering: Generating knowledge for action. Plos $M$ ed 2006; 3(4):425-8.

24. Geyman J. Health care in America. Can our ailing system be healed? Boston: Butterworth Heinemann; 2002.

25. Evidence-based medicine working group. Evidence-based medicine: a new approach to teaching the practice of medicine. JAMA 1992; 268:2420-5.

26. Tonelli MR. The philosophical limits of evidencebased medicine. Acad M ed 1998; 73(12):1234-40.

27. Lexchin J. Bigger and better: How Pfizer redefined erectile dysfunction. Plos M ed 2006; 3(4):429-32.

28. Tiefer L. Female sexual dysfunction: a case study of disease mongering and activist resistance. Plos M ed 2006; 3(4):436-40.

29. Healy D. The latest mania: Selling bipolar disorder. Plos M ed 2006; 3(4):441-4.

30. Phillips $C$. M edicine goes to school: teachers as sickness brokers for ADHD. Plos M ed 2006; 3(4):433-5.
31. Moynihan R, Cassels A. Selling sickness: how the world's biggest pharmaceutical companies are turning us all into patients. New York: Nation Books; 2005.

32. Angell M. The truth about the drug companies: how they deceive us and what to do about it. New York: Random House; 2004.

33. Critser G. Generation Rx. How prescription drugs are altering American lives, minds, and bodies. New York: Houghton M ifflin; 2005.

34. Applbaum K. Pharmaceutical marketing and the invention of the medical consumer. Plos M ed 2006; 3(4):445- 7

35. Lakoff A. The anxieties of globalization. Antidepressant sales and economic crisis in Argentina. Social Studies of Science 2004; 34(2):247-269.

36. Mintzes $B$. Disease mongering in drug promotion: Do governments have a regulatory role? Plos $\mathrm{M}$ ed 2006; 3(4):461-5.

37. Fitzpatrick M. The tyranny of health. Doctors and the regulation of lifestyle. London \& New York: Routledge; 2001.

Artigo apresentado em 07/02/2008

Aprovado em 12/03/2008 